\title{
Survey on Congestion Elusion Topography in Wireless Sensor Network using K-Map
}

\author{
Ayesha Taranum ${ }^{1}$, Dr. Reshma Banu ${ }^{2}$ \\ Assistant Professor, Dept of ISE, GSSSIETW, Mysore ${ }^{1}$ \\ Professor \& HOD, ISE, GSSSIETW, Mysore ${ }^{2}$
}

\begin{abstract}
Wireless Sensor Network (WSN) is an ad-hoc net-work consisting of sensor nodes having limited computational power fitted with a radio transceiver. This has been enabled by the availability, particularly in recent years, of sensors that are smaller, cheaper, and intelligent. These sensors are equipped with wireless interfaces with which they can communicate with one another to form a network. The design of a WSN depends significantly on the application, and it must consider factors such as the environment, the application's design objectives, cost, hardware, and system constraints. Main constraint of a sensor node is the limitation of its energy resource in the form of a short battery life. Battery power conservation is very important in a sensor device since battery life is usually one of the critical components in extending the life time of sensor nodes and its network. Therefore energy optimization techniques must be performed while designing a network system. Problems of how to reduce energy consumption to maximize network lifetime has been studied extensively. But most of the approaches focus on selecting the shortest or energy-efficient routing path, often change in leader nodes based on residual energy, packet size optimization to prolong network lifetime. Work has been done on congestion control and minimization of packet Percussion, however congestion Elusion in sensor network has not received serious study until recently. The paper describes a network using congestion Elusion Topography or PETopography where every wireless sensor is deterministically deployed throughout the sensing area. A congestion Elusion data retrieval strategy is proposed by forming an optimal congestion Elusion tree or PETree of sensor nodes using K-map and K-graph. Network ensures congestion free multi-hop data routing that significantly reduces packet Percussion and retransmission. As a result sink packet loss ratio and network end-to-end delay improves and maximizes energy efficiency and network lifetime.
\end{abstract}

Index Terms: packet loss; packet retransmission, wireless sensor network; sensor nodes; stationary sink; network lifetime; energy efficiency; congestion detection; congestion Elusion.

\section{INTRODUCTION}

Wireless sensor network (WSN) is a field of great interest in industrial and scientific research because of the fact that it is different from traditional wireless networks and involves various emerging technologies. Any WSN is formed with a large number of spatially distributed nodes organize themselves in an ad-hoc fashion which can sense, compute and communicate to cooperatively monitor physical phenomenon or environmental conditions at different locations. Each node is battery operated and consumes energy during data transmission, data reception, processing query requests and forwarding queries/data to neighboring nodes. It is generally difficult to charge/replace exhausted batteries. Therefore low energy consumption and maximize node/network lifetime becomes a primary objective in WSNs design.

In wireless sensor networks hundreds or thousands of sensor nodes transmit packet about a physical phenomenon to a single or more sinks using multi-hop routing. Proactive networks send data periodically. Reactive networks operating under idle or light load, report data at a lower rate. Usually sensor receives more data than it can forward and excess data is buffered. But congestion occurs when buffer overflows and excessive packets have to be dropped. Moreover multi-hop nature of WSN additionally creates network congestion by increasing the level of local contention [10] and interference in the shared wireless medium of limited capacity. The main reason of congestion is sensor nodes that are allowed to transmit packets as many as they can which result in Percussions and packet corruption. Since corrupted packets must be retransmitted, Percussions increase latency and incur excess energy consumption. It is one of the major factor that reduce the overall energy efficiency of the network.

In general, congestion leads to Percussion between transmitted packets. Radio Percussion occurs when many sensors attempt to send data at the same time and over the same transmission medium or channel. Percussions in WSN has the following drawbacks:

- increase energy consumption of sensor nodes

- causes packet loss, which reduces the network throughput

- Percussion-induced packet retransmission 
- $\quad$ energy spent on the packet is wasted when it is dropped. More it has travelled, more the waste is

- $\quad$ hinders fair event detections and fails to provide reliable data transmissions

Intense study and extensive research has been conducted on physical, MAC and network layer. A cross-layer control strategy [7][2] that jointly considers routing at network layer and MAC layer retransmission is adopted to improve the network lifetime. However the problem of Percussion Elusion in WSN remains largely open. There are several transport protocols that have been designed for WSNs to consider congestion control and guarantees reliability but never discusses anything on how to prevent congestion [4] from happening.

\section{RELATED WORK AND LIMITATION}

High volume of data traffic is generated when an event is detected by sensors. It is the period when sensory information becomes the utmost important and the likelihood of conges-tion becomes the greatest. Accurate and efficient congestion detection and control is always a big challenge. In this section state of the art is discussed to address congestion problem that eventually jeopardize the energy-efficient WSN functionality.

\section{A. Reporting Rate-based Mechanism}

In WSN, most current congestion control mechanisms are slow-converging rate based control. But the difficulty is to determine the correct amount of rate reduction [3] by the upstream sensors in response to downstream congestion. On the other hand Additive Increase Multiplicative Decrease (AIMD) [13] relies on periodic rate adjustment. No mechanism exist which can allow sensors to quickly adapt their rates according to changes in environmental dynamics.

\section{B. Contention-based Mechanism}

In contention-based protocol, nodes always sense channel before transmission to detect any ongoing communication. The mechanism ensures Percussion Elusion near the sender but cannot solve the hidden node problem at receiver.

\section{Medium Access Control (MAC) Protocol}

Traditionally WSN attempts to solve Percussion problem in the MAC layer. MAC protocols assist nodes to decide when and how to access the channel. However making decision to access the medium within a dense network of nodes becomes a challenging problem. TDMA-based protocols impose a deterministic transmission schedule. However coordination among all sources is ensured through communication that induces extra latency and excess energy consumption to an already power constrained system. RTS/CTS handshake mechanism eliminates hidden terminal problem but introduces exposed node problem. Moreover RTS/CTS approach assumes sym-metric channels [9] and only applicable for P2P communications. Mechanism becomes infeasible in WSN where broadcast communication and presence of asymmetric links are very common.

\section{Transport Protocol}

Various energy efficient congestion control scheme for sensor networks are proposed at transport layer. Congestion Detection and Elusion (CODA) [11] protocol where each node detects congestion by monitoring the channel utilization and buffer occupancy level. On detection, it broadcasts back-pressure messages and signals are propagated through the up- stream nodes down towards the source so that neighbors may drop packets and source may reduce its sending rate. Closed-loop multi-source regulation comes into play when persistent congestion happens due-to many sources start reporting data at a rate greater than a pre-configured threshold. Sink sends continuous stream of ACKs in order to regulate the source rate. Fusion is another congestion control and notification protocol like CODA. It uses stop-andstart hop-by-hop rate adjustment mechanism but replaces explicit control packets with implicit ACK (IACK) that piggybacks congestion bit in the packet header. It allows prioritized access to the channel for congested nodes. Eventto-sink reliable transport (ESRT) [1] is a protocol both for congestion control and reliability. It guarantees event reliability in the upstream direction through end-to-end source rate adjustment. On the other hand Reliable MultiSegment Transport (RMTS) [8] and Reliable Bursty Con-vergecast (RBC) protocols provide packet reliability through hop-by-hop loss recovery mechanism. Sensor Transmission Control Protocol (STCP) [6] provides both. But all of the above protocols do not consider node priority and multi-path routing used in the network layer and rarely consider cross-layer interactions [12]. They work in reactive mode and do not prevent congestion to happen.

\section{CONGESTION ELUSION TOPOGRAPHY}

Congestion Elusion Topography or PETopography is a new wireless sensor network Topography. It proposes a congestion free multi-hop routing mechanism and an energy-aware data retrieval strategy. Sink exploits the new network Topography for sensor data collection. Following subsections discuss in details about Percussion Elusion network formation, routing methodology and sensor data gather operation. 


\section{A. Working Principle}

The work describes a novel strategy that states how to improve WSN lifetime by reducing energy consumption of power constrained sensor nodes. A new network Topography is defined where sensor nodes are deterministically deployed within the area of interest. Sink maintains neighbourhood relationships hierarchically among all upstream nodes in the form of a Karnaugh map of K-map based on node association request. The rule of pair is applied on the Kmap and a Karnaugh graph or K-graph of sensor nodes is created that represents the adjacency relationship. Connectivity among sensor nodes uses a tree data structure. It is a spanning tree formed from the K-graph. Classical depth-first-traversal is used in a controlled way to build the tree keeping sink as starting vertex. Algorithm ensures the tree formation in such a way that it will be free from link level data Percussion. Build mechanism optimizes the tree height. The newly formed tree is known as Optimal Percussion Elusion Tree or optimal PETree. Sensor data retrieval operates on a new congestion free multi-hop routing protocol. It reduces packet loss and packet error due to Percussion and thereby minimizes packet retransmission. Reduction of packet retransmisison eventually brings down average transmit energy consumption of sensor nodes to a greater extent. Hence improves the network lifetime.

\section{B. K-Map and K-Graph of Sensor Nodes}

This subsection provides details about the work to create a K-map of wireless sensor nodes. It discusses the method and technique regarding how to form a K-map of $\mathrm{N}$ sensor nodes. It also describes how to obtain K-graph from a K-map.

The conventional Karnaugh map or K-map is a technique to simplify Boolean algebraic expressions. The required Boolean results are transferred from a truth table onto a two-dimensional grid where the cells are ordered in grey code and each cell position represents one combination of input conditions. Again each cell value represents the corresponding output value. A k-variable $\mathrm{K}$-map contains $\mathrm{N}=2^{\mathrm{k}}$ minterms $\left(\mathrm{m}_{0}, \mathrm{~m}_{1}, \mathrm{~m}_{2}, \ldots, \mathrm{m}_{\mathrm{N}-1}\right)$ and the size of the Kmap is equal to the total number of minterms. But K-map of sensor nodes is somewhat different. A wireless sensor network comprising of sensor nodes, can be represented as a $\mathrm{k}$-variable $\mathrm{K}$-map that contains $\mathrm{N}$ sensor nodes and $\mathrm{k}=$ $\operatorname{lgN}$. The size of the K-map becomes the network size $\mathrm{N}$ and minterms become the sensor members. It is a diagram consisting of a rectangular array of squares where each cell represents a different sensor node. Cells are occupied based on node association request with a sink. The way K-map rules of pair to group adjacent cells are applied to simplify Boolean expressions; same rules can be used to determine node adjacency information in a network. The advantage of a $\mathrm{K}$-map is, if a particular node is selected and nodes belong to its adjacent cells are grouped, all nearby nodes can be determined. Rules evaluate that each node $\mathrm{i} \in \mathrm{N}$ of a $\mathrm{k}$-variable $\mathrm{K}$-map can have at most $\mathrm{k}$ number of neighbour nodes and node degree becomes $\mathrm{k}$. If $\forall \mathrm{i} \in \mathrm{N}$, node degree $\mathrm{d}(\mathrm{i})=\mathrm{k}$, K-graph transforms into k-regular K-graph. Node adjacency information prepares node adjacency list to form a K-graph of $\mathrm{N}$ sensor nodes.

A simple algorithm is devised to obtain adjacency list from K-map. Algorithm [5] uses the generic expression based on rule of pair and for a given cell value $\mathrm{i}$ calculates all other nearby node positions.

$$
\left.\mathrm{i}+\left((-1)^{\mathrm{n}}\right) 2^{\mathrm{k}}\right) \text { where } \mathrm{n}=\mathrm{i} / 2^{\mathrm{k}}
$$

TABLE I ADJACENCY LIST OF 4 NODES

\begin{tabular}{|c|c|}
\hline Node & Adjacency Relationship \\
\hline 0 & $1 \rightarrow 2 \rightarrow$ N U LL \\
\hline 1 & $0 \rightarrow 3 \rightarrow$ N UL \\
\hline 2 & $0 \rightarrow 3 \rightarrow$ N U LL \\
\hline 3 & $1 \rightarrow 2 \rightarrow$ N U LL \\
\hline
\end{tabular}

TABLE II ADJACENCY LIST OF 8 NODES

\begin{tabular}{|c|l|}
\hline Node & Adjacency Relationship \\
\hline 0 & $1 \rightarrow 2 \rightarrow 4 \rightarrow \mathrm{N}$ UL \\
\hline 1 & $0 \rightarrow 3 \rightarrow 5 \rightarrow \mathrm{N}$ UL \\
\hline 2 & $0 \rightarrow 3 \rightarrow 6 \rightarrow \mathrm{N}$ UL \\
\hline 3 & $1 \rightarrow 2 \rightarrow 7 \rightarrow \mathrm{N}$ UL \\
\hline 4 & $0 \rightarrow 5 \rightarrow 6 \rightarrow \mathrm{N}$ UL \\
\hline 5 & $1 \rightarrow 4 \rightarrow 7 \rightarrow \mathrm{N}$ UL \\
\hline 6 & $2 \rightarrow 4 \rightarrow 7 \rightarrow \mathrm{N}$ U LL \\
\hline 7 & $3 \rightarrow 5 \rightarrow 6 \rightarrow \mathrm{N}$ U LL \\
\hline
\end{tabular}


TABLE III ADJACENCY LIST OF 16 NODES

\begin{tabular}{|c|c|}
\hline Node & Adjacency Relationship \\
\hline 0 & $1 \rightarrow 2 \rightarrow 4 \rightarrow 8 \rightarrow \mathrm{N}$ UL \\
\hline 1 & $0 \rightarrow 3 \rightarrow 5 \rightarrow 9 \rightarrow \mathrm{N}$ U LL \\
\hline 2 & $0 \rightarrow 3 \rightarrow 6 \rightarrow 10 \rightarrow \mathrm{N}$ UL \\
\hline 3 & $1 \rightarrow 2 \rightarrow 7 \rightarrow 11 \rightarrow \mathrm{N}$ UL \\
\hline 4 & $0 \rightarrow 5 \rightarrow 6 \rightarrow 12 \rightarrow \mathrm{N} \mathrm{U} \mathrm{LL}$ \\
\hline 5 & $1 \rightarrow 4 \rightarrow 7 \rightarrow 13 \rightarrow \mathrm{N}$ UL \\
\hline 6 & $2 \rightarrow 4 \rightarrow 7 \rightarrow 14 \rightarrow \mathrm{N} \mathrm{U}$ LL \\
\hline 7 & $3 \rightarrow 5 \rightarrow 6 \rightarrow 15 \rightarrow \mathrm{N}$ LL \\
\hline 8 & $0 \rightarrow 9 \rightarrow 10 \rightarrow 12 \rightarrow \mathrm{N}$ UL \\
\hline 9 & $1 \rightarrow 8 \rightarrow 11 \rightarrow 13 \rightarrow \mathrm{N}$ UL \\
\hline 10 & $2 \rightarrow 8 \rightarrow 11 \rightarrow 14 \rightarrow \mathrm{N}$ UL \\
\hline 11 & $3 \rightarrow 9 \rightarrow 10 \rightarrow 15 \rightarrow \mathrm{N}$ UL \\
\hline 12 & $4 \rightarrow 8 \rightarrow 13 \rightarrow 14 \rightarrow \mathrm{N}$ ULL \\
\hline 13 & $5 \rightarrow 9 \rightarrow 12 \rightarrow 15 \rightarrow \mathrm{N}$ UL \\
\hline 14 & $6 \rightarrow 10 \rightarrow 12 \rightarrow 15 \rightarrow \mathrm{N}$ UL \\
\hline 15 & $7 \rightarrow 11 \rightarrow 13 \rightarrow 14 \rightarrow \mathrm{N}$ LL \\
\hline
\end{tabular}

A sink maintains all upstream nodes neirgbourhood relationship in the form of a K-map. Figures 1(a),1(b),1(c) show Kmaps with 4,8 and 16 nodes respectively, where sink is the root node $\mathrm{N} 0$ and occupies cell- 0 position. According to Fig.1 (a) if node $\mathrm{N} 0$ is selected and by the virtue of $\mathrm{K}$-map definition, nodes belong to adjacent cells are grouped, three pair of nodes $(\mathrm{N} 0, \mathrm{~N} 1),(\mathrm{N} 0, \mathrm{~N} 2)$ and $(\mathrm{N} 0, \mathrm{~N} 4)$ are formed. They are all neighbours of each other. Similarly for Figures 1(b),and 1(c) apply the same working principle and obtain four pair of nodes ( $\mathrm{N} 0, \mathrm{~N} 1),(\mathrm{N} 0, \mathrm{~N} 2)(\mathrm{N} 0, \mathrm{~N} 4)$ and ( $\mathrm{N} \mathrm{0,N} \mathrm{8)} \mathrm{who} \mathrm{are} \mathrm{all} \mathrm{nearby} \mathrm{nodes} \mathrm{of} \mathrm{each} \mathrm{other.} \mathrm{Figures} \mathrm{2(a),} \mathrm{2(b),} \mathrm{2(c)} \mathrm{show} \mathrm{K-graphs} \mathrm{and} \mathrm{Tables} \mathrm{I,} \mathrm{II,} \mathrm{III}$ represent adjacency lists of 4, 8 and 16 sensor nodes. K-map is a recursive structure and can be applied for higher nodes ( $\mathrm{N} \gg$ 16) recursively. Similarly the same procedure can be used further to prepare K-graphs from K-maps of higher nodes without any algorithimic modifications.

\section{Percussion Elusion Tree (PETree)}

This subsection provides details about the work to create optimal PETree from a K-graph of N sensor nodes. PETree is a new tree data structure.

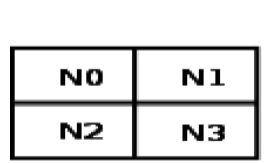

(a) K-map of 4 Nodes

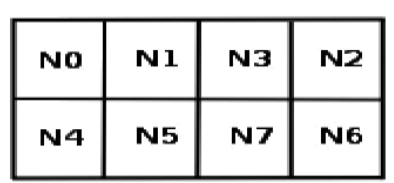

(b) K-map of 8 Nodes

\begin{tabular}{|l|l|l|l|}
\hline No & $N 1$ & $N 3$ & $N 2$ \\
\hline$N 4$ & $N 5$ & $N 7$ & $N 6$ \\
\hline$N 12$ & $N 13$ & $N 15$ & $N 14$ \\
\hline$N 8$ & $N 9$ & $N 11$ & $N 10$ \\
\hline
\end{tabular}

(c) K-map of 16 Nodes

Fig. 1. K-map of Wireless Sensor Nodes

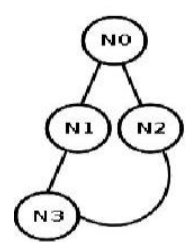

(a) K-graph of 4 Nodes

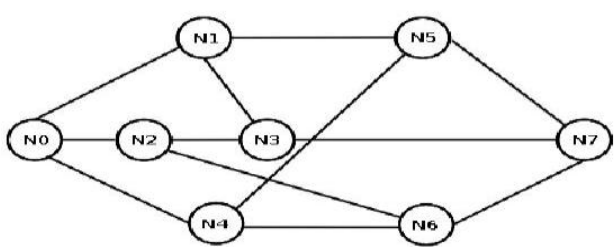

(b) K-graph of 8 Nodes

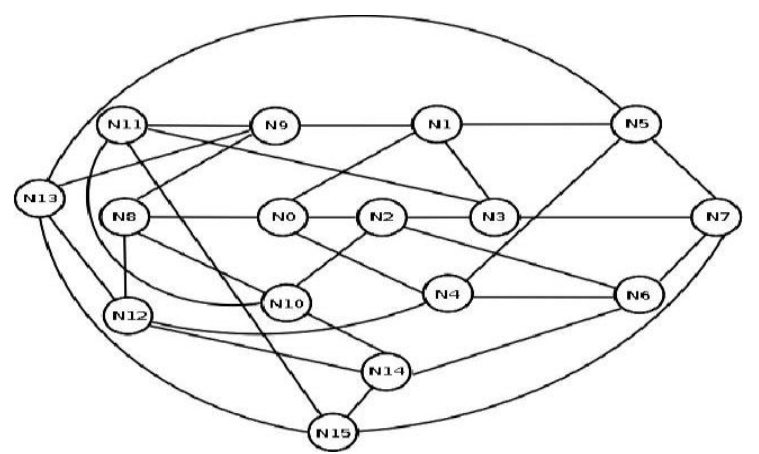

(c) K-graph of 16 Nodes

Fig. 2. K-graph of Wireless Sensor Nodes 
As the name suggests Percussion Elusion, it implies that it is a tree rooted with a sink and can gather sensory data from its entire successor nodes by completely avoiding branch/link level congestion. According to graph theory PETree appears like a k-array or k-way tree in which each node shouldn't have more than $\mathrm{k}$ children. The value of $\mathrm{k}$ is the size of a k-variable K-map contains $\mathrm{N}$ sensor nodes. Maximum degree of each node can also reach $\operatorname{lgN}$. K-graph is used to build the tree. Depth-first-traversal is applied on K-graph to obtain a spanning tree. Set of rules are then applied to satisfy constraints in order to arrive a PETree with optimal height. The oprtimal height of PETree of $\mathrm{N}$ nodes is $\operatorname{lgN}$. In the following subsections properties of a PETree and its formation from a K-graph of $\mathrm{N}$ sensor nodes is discussed.

The aim of the sink is to form a tree of $\mathrm{N}$ sensor nodes from K-graph with an optimal height which should be free from data congestion at link level. That's what a Percussion Elusion tree is. Figures 3(a), 3(b), 3(c) show PETree with 4, 8 and 16 nodes respectively. In all cases sink is the root node N 0. In Fig.3(a) the nodes N 2 and N 3 are the leaves, while in Fig.3(b) and Fig.3(c) the nodes N 2, N 5, N 6, N 7 and N 4, N 9, N 11, N 12, N 13, N 14, N 15 are the leaves. Each node is associated with a level l, as marked along the edges above the corresponding node as shown in Figures of PETree. In Fig.3(a) N 2, N 3 are level 2 nodes and $\mathrm{N} 1$ is a level 1 node whereas in Fig.3(b) N 2, N 5, N 6, N 7 are level 3 nodes, N 3, N 4 are level 2 nodes and N 1 is a level 1 node and in Fig.3(c) N 4, N 9, N 11, N 12, N 13, N 14, N 15 are level 5 nodes, $\mathrm{N}$ 5, N 6, N 7, N 10 are level 4 nodes, N 2, N 8 are level 3 nodes, and finally N 3 and $\mathrm{N} 1$ are level 2 and level 1 nodes respectively.

A PETree of $\mathrm{N}$ nodes has the following properties:

- $\quad$ each node of a PETree is associated with a level 1

- root node has a level $1=0$ and the subsequent off-springs have levels $1=1,2, \ldots \mathrm{n}$ where $\mathrm{n}=\operatorname{lgN}$. The level of a node denotes the communication round during which the node transmits data if a gather operation is performed with the Percussion Elusion tree to which the node belongs.

- $\quad$ each edge in the PETree represents a data transfer be-tween two nodes of different levels.

- all index arithmetic is assumed to be modN. Thus no, two leaves from a single parent are at the same level. This rule ensures that there is no Percussion in any communication round whereas Percussion occurs among two nodes trying to send data to a single node at the same instant using star, mesh or cluster tree Topography.

- data transfer is initiated from the leaves of the PETree to the root for a gather operation. Nodes belonging to a particular level transmit data simultaneously to the nodes of a preceding level constitute a communication round. Since there are $\mathrm{n}$ different levels in a PETree the complete data transfer takes communication $\operatorname{lgN}$ rounds.

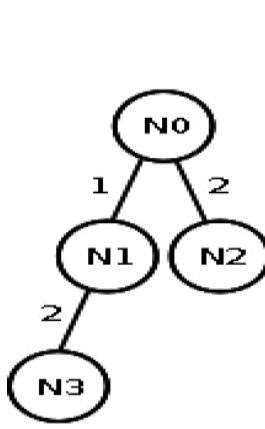

(a) PETree of 4 Nodes

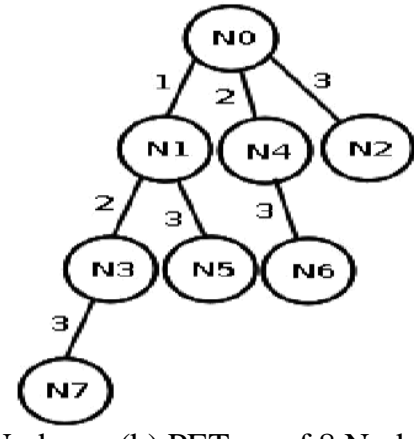

(b) PETree of 8 Nodes

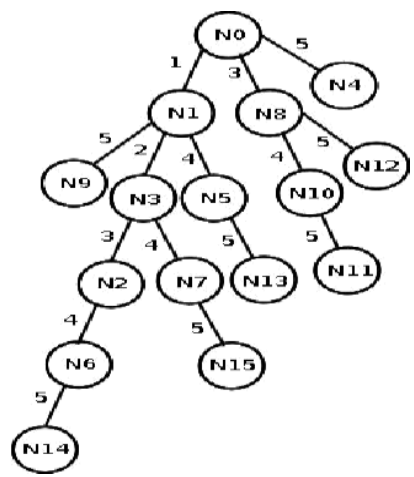

(c) PETree of 16 Nodes

Fig. 3. PETree of Wireless Sensor Nodes

- in a scatter operation root broadcasts data request to the child nodes and subsequently the message is forwarded to succeeding nodes along the edges of a PETree. Similar to gather operation broadcast message takes transfers to arrive all nodes. Data retrieval starts only when each node receives the message. On gather operation nodes transmit the complete data set of its entire offspring to its parent and finally reach to the root node.

The advantages of the PETree therefore, are the followings:

- $\quad$ gather operation takes $=\operatorname{lgN}$ communication rounds, instead of $\mathrm{N}$ rounds which would have been the case had the data elements been gathered sequentially.

- Topography of the PETree ensures that simultaneous com-munication occurs only along disjoint edges and hence, in cases where it is possible to partition the network using clusters, for example there would be no Percussion if multiple cluster trees are formed. Thus, the latency would remain unaltered. This is particularly important for wireless sensor network applications. 
D. Congestion Free Routing

Percussion is an unavoidable phenomenon happens in any type of wireless routing. Result is retransmission. Problem is more severe in mesh routing. Tree routing suffers from link congestion.

Name of the protocol implies a Percussion free routing. Pro-posed strategy takes the inherent advantage of a new data structure PETree to arrest congestion happen during data gather operation. Sink transmits data request packets which are similar to beacon messages to upstream nodes functioning as transponders. The data request packet causes the transponder to reply by sending a large number of data packets to the sink.

Protocol works as follows:

- $\quad$ starts to operate on receipt of a beacon message send by the sink

- nodes are at same level send data in parallel

- total number of rounds needed to finish data gathers is $\operatorname{lgN}$

TABLE IV SCATTER OPERATION AMONG 4 NODES

\begin{tabular}{|c|c|}
\hline Message Transfer Rounds & Scatter Operation \\
\hline 1 & $\{\mathrm{~N} 0 \rightarrow \mathrm{N} 1\},\{\mathrm{N} 0 \rightarrow \mathrm{N} 2\}$ \\
\hline 2 & $2\{\mathrm{~N} 1 \rightarrow \mathrm{N} 3\}$ \\
\hline
\end{tabular}

TABLE V GATHER OPERATION AMONG 4 NODES

\begin{tabular}{|c|c|}
\hline Data Transfer Rounds & Gather Operation \\
\hline 1 & $\{\mathrm{~N} 3 \rightarrow \mathrm{N} 1\},\{\mathrm{N} 2 \rightarrow \mathrm{N} \mathrm{0}\}$ \\
\hline 2 & $\{\mathrm{~N} 1 \rightarrow \mathrm{N} 0\}$ \\
\hline
\end{tabular}

TABLE VI SCATTER OPERATION AMONG 8 NODES

\begin{tabular}{|l|l|}
\hline Message Transfer Rounds & Forward of Data Request Packet (Scatter Operation) \\
\hline 1 & $\{\mathrm{~N} 0 \rightarrow \mathrm{N} 1\},\{\mathrm{N} 0 \rightarrow \mathrm{N} 2\},\{\mathrm{N} 0 \rightarrow \mathrm{N} 4\}$ \\
\hline 2 & $\{\mathrm{~N} 1 \rightarrow \mathrm{N} 3\},\{\mathrm{N} 1 \rightarrow \mathrm{N} \mathrm{5}\},\{\mathrm{N} 4 \rightarrow \mathrm{N} 6\}$ \\
\hline 3 & $\{\mathrm{~N} 3 \rightarrow \mathrm{N} 7\}$ \\
\hline
\end{tabular}

Tables IV, V, VI, VII, VIII, IX show how data request packets reach to each nodes or transponders and congestion free routing takes place along the path of a PETree during data gather operation. Scatter and gather operation happens during data retrieval between groups of sensor nodes.

TABLE VII GATHER OPERATION AMONG 8 NODES

\begin{tabular}{|c|c|}
\hline Data Transfer Rounds & Parallel Flow of Sensory Data(Gather Operation) \\
\hline 1 & $\{N 7 \rightarrow N 3\},\{N 5 \rightarrow N 1\},\{N 6 \rightarrow N 4\},\{N 2 \rightarrow N 0\}$ \\
\hline 2 & $N 3 \rightarrow N 1\},\{N 4 \rightarrow N 0\}$ \\
\hline 3 & $\{N 1 \rightarrow N 0\}$ \\
\hline
\end{tabular}

TABLE VIII SCATTER OPERATION AMONG 16 NODES

\begin{tabular}{|c|c|}
\hline Message Transfer Rounds & Forward of Data Request Packet (Scatter Operation) \\
\hline 1 & $N 0 \rightarrow N 1\},\{N 0 \rightarrow N 4\},\{N 0 \rightarrow N 8\}$ \\
\hline 2 & $\{N 1 \rightarrow N 3\},\{N 1 \rightarrow N 5\},\{N 1 \rightarrow N 9\},\{N 8 \rightarrow N 10\},\{N 8 \rightarrow N 12\}$ \\
\hline 3 & $\{N 3 \rightarrow N 2\},\{N 3 \rightarrow N 7\},\{N 5 \rightarrow N 13\}\{N 10 \rightarrow N 11\}$ \\
\hline 4 & $\{N 2 \rightarrow N 6\},\{N 7 \rightarrow N 15\}$ \\
\hline 5 & $\{N 6 \rightarrow N 14\}$ \\
\hline
\end{tabular}

E. Estimation of Data Retrieval Time

if each transfer takes td seconds for a single packet, the total data transfer time for the gather part Tg from all nodes and send to sink can be written as:

$$
\operatorname{Tg}=(\lg N)(\mathrm{td})
$$


above expression can be re-written as:

$$
\operatorname{Tg}=\text { ntd }
$$

where $\mathrm{N}$ is the number of wireless sensor nodes and $\mathrm{n}$ is the total number of node levels in a scatter operation data request is broadcast by the sink and message is received by each node. Again if each transfer takes td seconds for a single broadcast packet, the total message transfer time for the scatter part Ts nodes from sink can be written as:

$$
\mathrm{Ts}=(\operatorname{lgN})(\mathrm{td})=\mathrm{ntd}
$$

time required for broadcasting the request message followed by sensor data retrieval can be written as:

$$
\mathrm{T}=2 \mathrm{ntd}=2(\operatorname{lgN})(\mathrm{td})
$$

if the message for data request can be broadcast to all

$$
\mathrm{T}=\mathrm{td}+\mathrm{ntd}=(\mathrm{n}+1) \mathrm{td}=(\operatorname{lgN}+1) \mathrm{td}
$$

\section{SIMULATION AND RESULTS}

OPNET is used to simulate a ZigBee wireless sensor network. OPNET contains device models for ZIgBee coordinators, routers and end devices. Three hierarchical levels for configuration are differentiated. Brief overview of the simulator used:

- network level creates the Topography of the network under investigation

- node level defines the behavior of the node and control the flow of data between different functional elements inside the node

- process level describing the underlying protocols are represented by FSMs and created with states and statetransitions

\begin{tabular}{|c|c|}
\hline Data Transfer Rounds & Parallel Flow of Sensory Data(Gather Operation) \\
\hline 1 & $\begin{aligned}\{\mathrm{N} 9 & \rightarrow \mathrm{N} 1\},\{\mathrm{N} 14 \rightarrow \mathrm{N} 6\},\{\mathrm{N} 15 \rightarrow \mathrm{N} 7\},\{\mathrm{N} 13 \rightarrow \mathrm{N} 5\} \\
& \{\mathrm{N} 11 \rightarrow \mathrm{N} 10\},\{\mathrm{N} 12 \rightarrow \mathrm{N} 8\},\{\mathrm{N} 4 \rightarrow \mathrm{N} 0\} \rightarrow\end{aligned}$ \\
\hline 2 & $\{\mathrm{~N} 6 \rightarrow \mathrm{N} 2\},\{\mathrm{N} 7 \rightarrow \mathrm{N} 3\},\{\mathrm{N} 5 \rightarrow \mathrm{N} 1\},\{\mathrm{N} 10 \rightarrow \mathrm{N} 8\}$ \\
\hline 3 & $\{\mathrm{~N} 2 \rightarrow \mathrm{N} 3\},\{\mathrm{N} 8 \rightarrow \mathrm{N} 0\}$ \\
\hline 4 & $\{\mathrm{~N} 3 \rightarrow \mathrm{N} 1\}$ \\
\hline 5 & $\{\mathrm{~N} 1 \rightarrow \mathrm{N} 0\}$ \\
\hline
\end{tabular}

The source code is based on $\mathrm{C} / \mathrm{C}++$. The analysis of simulated data is supported by a variety of built-in functions. A different graphical presentation for the simulation results exists.

\section{TABLE IX GATHER OPERATION AMONG 16 NODES}

\section{A. Experimental Results}

The main focus of the network simulation is to analyze network performance in the context of a WSN. Network simulator OPNET has been used to implement PETopography sensor nodes and to measure the performance of data retrieving strategy using a sink. Four scenarios have been considered and tested in the simulator, version 14.5 with wireless package.

Model implements ZigBee application and network protocol on top of IEEE 802.15.4 PHY and MAC layers. In all four test scenarios, sink and all other sensor nodes are stationary. Nodes $\mathrm{N}=60$ are uniformly deployed through out the grid of medium $100 \times 100$ scale. In the first scenario network is a Star Topography. A proper sink placement is examined so that each node will be at 1-hop distance and can send data directly using AODV protocol. In the second and third scenario, network is a Mesh and Tree Topography and

- $\quad$ sensor node distribution : uniform

- $\quad$ receiver sensitivity : $-90 \mathrm{dbm}$

- $\quad$ packet size : 1024 bytes

- $\quad$ packet generation interval : $0.5 \mathrm{sec}$

- data rate $: 250 \mathrm{kbps}$

- $\quad$ no of nodes : 60 
Vol. 6, Issue 4, April 2017

TABLE X ZIGBEE PARAMETERS

\begin{tabular}{|c|c|c|c|}
\hline Parameters & Star & Mesh & Tree \\
\hline Max.childrens & 255 & 3 & 3 \\
\hline Max.routers & 0 & 2 & 2 \\
\hline Max.depth & 1 & 5 & 5 \\
\hline Mesh routing & Disabled & Disabled & Disabled \\
\hline Routing protocol & AODV & AODV & Tree \\
\hline Transmit power & 0.05 & 0.05 & 0.05 \\
\hline Transmit band & $2.4 \mathrm{GHz}$ & $2.4 \mathrm{GHz}$ & $2.4 \mathrm{GHz}$ \\
\hline ACK mechanism & Enable & Enable & Enable \\
\hline
\end{tabular}

Perfromance evaluation of four test scenarios is conducted on the following metrics captured from OPNET statistics:

- $\quad$ network end-to-end delay

- $\quad$ sink bit error rate

- $\quad$ sink packet loss ratio

- $\quad$ sink bit errors per packet B. Evaluation and Discussion

PETopography outperforms star, mesh and tree network with a static sink in network end-to-end delay, sink bit error rate, bit error per packet and packet loss ratio. Moreover significant improvement has been achieved in bit error per packet and packet loss ratio. Congestion free routing ensures two packets cannot reach a point in the network at the same time and avoid packet Percussion. Packets are not dropped by the network. As a result packet retransmissions are reduced and network end-to-end delay improves. Hence in all aspects PETopography can be thought off to use in order to maximize WSN lifetime.

\section{CONCLUSION}

A network Topography is described to maximize the life-time of a WSN by minimizing energy consumption of power constrained sensor nodes using a stationary sink. Network is created using PETopography where every wireless sensors are deterministically deployed throughout the sensing area. A congestion Elusion data retrieval strategy is proposed by forming an optimal PETree network of sensor nodes using K-map and K-graph. Implicit network structure ensures congestion free multi-hop data routing that significantly reduces packet Percussion and retransmission.

\section{REFERENCES}

[1] F. Bouabdallah, N. Bouabdallah, and R. Boutaba. Cross-layer design for energy conservation in wireless sensor networks. In Communications, 2009. ICC '09. IEEE International Conference on, pages 1-6, 2009.

[3] S. Brahma, M. Chatterjee, and K. Kwiat. "congestion control and fairness in wireless sensor networks". In Pervasive Computing and Communications Workshops (PERCOM Workshops), 2010 8th IEEE International Conference on, 2010.

[4] S. Chen and N. Yang. Congestion Elusion based on lightweight buffer management in sensor networks. Parallel and Distrinuted Systems, IEEE Transactions on, 17(9):934-946, 2006.

[5] A. Gupta and D. Ghosh. Karnaugh maps - an artificial intelligence approach. ACE '90. Proceedings of [XVI Annual Convention and Exhibition of the IEEE In India, pages 12-13, 1990.

[6] Y. Iyer, S. Gandham, and S. Venkatesan. Stcp: a generic transport layer protocol for wireless sensor networks. Computer Communications and Networks, 2005. ICCCN 2005. Proceedings. 14th International Conference on, 1(1-2):449-454, 2005.

[7] H. Kwon, T. H. Kim, S. Choi, and B. G. Lee. A cross-layer strategy for energy-efficient reliable delivery in wireless sensor networks. Wireless Communications, IEEE Transactions on, 5(12):3689-3699, 2006.

[8] F. Stann and J. Heidemann. Rmst: reliable data transport in sensor net-works. In Sensor Network Protocols and Applications, 2003. Proceedings of the First IEEE. 2003 IEEE International Workshop on, pages 102- 112, 2003.

[9] T. Stathopoulos, R. Kapur, D. Estrin, J. Heidemann, and L. Zhang. Application-based Percussion Elusion in wireless sensor networks. In Local Computer Networks, 2004. 29th Annual IEEE International Con-ference on, pages 506-514, 2004.

[10] M. C. Vuran, V. C. Gungor, and O. B. Akan. On the interdependence of congestion and contention in wireless sensor networks. In In Proceedings of the SenMetrics05, 2005.

[11] C.-Y. Wan, S. B. Eisenman, and A. T. Campbell. Coda: Congestion detection and Elusion in sensor networks. Proceedings of the 1st International Conference on Embedded Networked Sensor Systems, SenSys, (14):266-279, 2003.

[12] C. Wang, K. Sohraby, B. Li, M. Daneshmand, and Y. Hu. A survey of transport protocols for wireless sensor networks. Network, IEEE, 20(3):34-40, 2006.

[13] Y. Yang and S. Lam. "general aimd congestion control". In Network Protocols, 2000. Proceedings. 2000 International Conference on, pages 187-198, 2000.

[14] O. Akan and I. Akyildiz. Event-to-sink reliable transport in wireless sensor networks. Networking, IEEE/ACM Transactions on, 13(5):1003$1016,2005$. 